\title{
The Importance of Planetary Rotation Period for Ocean Heat Transport
}

\author{
J. Cullum, ${ }^{1,2}$ D. Stevens, ${ }^{1,2}$ and M. Joshi, ${ }^{1,3}$
}

\begin{abstract}
The climate and, hence, potential habitability of a planet crucially depends on how its atmospheric and ocean circulation transports heat from warmer to cooler regions. However, previous studies of planetary climate have concentrated on modeling the dynamics of atmospheres, while dramatically simplifying the treatment of oceans, which neglects or misrepresents the effect of the ocean in the total heat transport. Even the majority of studies with a dynamic ocean have used a simple so-called aquaplanet that has no continental barriers, which is a configuration that dramatically changes the ocean dynamics. Here, the significance of the response of poleward ocean heat transport to planetary rotation period is shown with a simple meridional barrier - the simplest representation of any continental configuration. The poleward ocean heat transport increases significantly as the planetary rotation period is increased. The peak heat transport more than doubles when the rotation period is increased by a factor of ten. There are also significant changes to ocean temperature at depth, with implications for the carbon cycle. There is strong agreement between the model results and a scale analysis of the governing equations. This result highlights the importance of both planetary rotation period and the ocean circulation when considering planetary habitability. Key Words: Exoplanet-Oceans-Rotation-Climate-Habitability. Astrobiology 14, 645-650.
\end{abstract}

\section{Introduction}

$\mathbf{T}$ HE NUMBER and rate of exoplanet discoveries is rapidly increasing (Akeson et al., 2013; Impey, 2013). Such discoveries motivate the question of the habitability of these alien worlds, which critically depends upon their climates (Kasting et al., 1993; Heller and Armstrong, 2014). Previous studies have investigated this question predominantly through modeling of the atmospheric component of the climate system, with only little consideration of the ocean. For instance, existing studies range from those with no ocean component (Joshi et al., 1997) to those that include a heavily simplified ocean, for example, a nondynamic slab ocean (Merlis and Schneider, 2010; Edson et al., 2011; Shields et al., 2013). The majority of studies that have considered a dynamic ocean have removed all continental barriers (Hu and Yang, 2013), which makes a dramatic difference to the dynamics and thermal structure of the ocean (Smith et al., 2006). The simplest way to model the dynamics that result from any continental configuration is to include a full-depth meridional barrier. It is clearly valid to expect the rotation period of planets to differ from that of Earth.
Del Genio and Suozzo (1987) considered the impact of different planetary rotation periods on the dynamics of planetary atmospheres. As part of this study, the poleward heat transport in the atmosphere, over a wide range of rotation periods, is presented in terms of the different components of the atmospheric heat transport; only small changes in magnitude are found when changing the rotation period. The peak contribution to poleward heat transport from the meridional circulation in each hemisphere has no discernible trend with rotation and varies by less than $2 \mathrm{PW}(1 \mathrm{PW}=$ $10^{15} \mathrm{~W}$ ) as the rotation period increases by three orders of magnitude. Williams (1988) also investigated this topic and concluded that as the rotation period increases the Hadley cell extends farther poleward, from $30^{\circ} \mathrm{N} / \mathrm{S}$ to $60^{\circ} \mathrm{N} / \mathrm{S}$ when increasing the rotation period from 1 to 8 days. The results from this study also show little trend in the response of the atmospheric heat transport to rotation period; over the range of rotation periods between 1 and 16 days, the high-latitude temperatures change by up to approximately $30^{\circ} \mathrm{C}$ but show no general relation to the rotation. A similar analysis in the study of the early Archean climate concludes a decrease in global surface air temperatures with a decrease in rotation

\footnotetext{
${ }^{1}$ Centre for Ocean and Atmospheric Sciences, ${ }^{2}$ School of Mathematics, and ${ }^{3}$ School of Environmental Sciences, University of East Anglia, Norwich, UK.
} 
period (Kiernet et al., 2013); however, this is over a significantly smaller range of rotation periods due to the application.

Vallis and Farneti (2009) investigated the effect of rotation period on the total (atmospheric and ocean) meridional energy transport as part of a wider study of the drivers of the atmosphere-ocean energy transport on Earth. The authors found a decrease in the total energy transport as the rotation period decreases. The atmospheric energy transport is found to have little dependence on rotation period, which agrees with the conclusions of Del Genio and Suozzo (1987) and Williams (1988). Vallis and Farneti (2009) found that the meridional ocean energy transport, when atmospheric and buoyancy forcing is included, increases as the rotation period is lengthened.

The ocean constitutes a substantial part of the climate system; on Earth, the heat transport by the atmosphere and ocean peaks at $5 \times 10^{15} \mathrm{~W}$ and $2 \times 10^{15} \mathrm{~W}$, respectively (Trenberth and Caron, 2001). In the present study, an analysis of the ocean dynamics was conducted, and a strong dependence of the poleward ocean heat transport on rotation period was shown. The results presented here add to the conclusions of Vallis and Farneti (2009) by including further details of the response of the ocean to rotation period. In particular, the change in the temperature structure of the ocean is clearly shown as part of this analysis. In addition, the present study isolates the response of the buoyancyforced component of the ocean heat transport from the additional forcing by the atmosphere. The consideration of this dependence of the ocean is required for a more complete conclusion of the impact of rotation period on global heat transport.

\section{Model and Methods}

This study employs the MOMA code (Webb, 1996), run at a resolution of $4^{\circ}$ in the horizontal and 15 vertical levels, which range in thickness from $30 \mathrm{~m}$ at the surface to $836 \mathrm{~m}$ at depth. This model allows the required flexibility for studies of different planetary configurations and has previously been applied in a similar way, though in an early Earth scenario, by Smith and Dubois (2004). All model runs have a horizontal momentum viscosity of $1 \times 10^{5} \mathrm{~m}^{2} \mathrm{~s}^{-1}$, vertical viscosity of $10^{-4} \mathrm{~m}^{2} \mathrm{~s}^{-1}$, and a horizontal and vertical diffusivity of $2 \times 10^{3} \mathrm{~m}^{2} \mathrm{~s}^{-1}$ and $10^{-4} \mathrm{~m}^{2} \mathrm{~s}^{-1}$, respectively. The surface momentum forcing, temperature, and salinity are based on corresponding zonally averaged climatologies of Hellerman and Rosenstein (1983) and Levitus (1982). Therefore, there is an implicit assumption that the stellar insolation is similar to that received by Earth.

Here, the results from five different ocean model runs are presented. The land configuration is either as Earth or a single, full-depth meridional barrier. Such a configuration is the simplest representation of any realistic continental distribution. In addition, it is the simplest configuration that maintains zonal pressure gradients and hence geostrophy, the dominant balance in the oceans on Earth (Smith et al., 2006). The meridional barrier connects two polar islands extending to $68^{\circ} \mathrm{N} / \mathrm{S}$, which are required to avoid grid cell convergence. When removing any zonal barrier to the flow, the role of the relatively small-scale eddy field becomes crucial, as is seen in the Antarctic Circumpolar Current (Hallberg and Gnanadesikan, 2006). The meridional heat transport would then be dominated by eddy transport, which is unfeasible to resolve for sensitivity studies such as this and is difficult to accurately parameterize (Hallberg and Gnanadesikan, 2006). In MOMA, eddy transports are parameterized by horizontal diffusion, which has been shown to be an acceptable parameterization in low-resolution ocean models at the rotation period of Earth (Smith et al., 2006). However, while eddy transports are expected to be dependent on rotation period, the eddy parameterization is not, which implies that ocean heat transports in aquaplanet models with no barriers might not be accurately represented without addressing this issue.

When the land configuration is changed to an aquaplanet with a meridional boundary, which we refer to as a ridgeplanet, the surface forcing is set to be hemispherically symmetric. The surface wind stress, temperature, and salinity forcings are made symmetrical by reflecting the southern hemisphere forcing in the northern hemisphere. The runs are forced by either buoyancy differences and wind, or buoyancy alone; for the solely buoyancy-driven runs, the wind stress is simply set to zero at all latitudes. To achieve meaningful results when lengthening the rotation period, the wind forcing is removed, as it is known that the winds are sensitive to rotation period (Del Genio and Suozzo, 1987; Williams, 1988). Hence, removing the wind forcing and maintaining a meridional barrier allows the clearest conclusions to be made about the impact of planetary rotation period on ocean heat transport.

The rotation period in the runs is either 1, 3, or 10 Earth days. The first run is as Earth, then the land configuration is changed to a barrier for the next run, then wind forcing is removed, and finally the rotation period is increased to 3 Earth days and 10 Earth days. In each case, the model runs for 8000 years, by which time the kinetic energy and ocean heat transport are effectively steady, so that they have changed by less than $0.1 \%$ over 1000 years. The results presented are an average of the last 1000 years of model output, at intervals of 100 years.

\section{Results}

As the rotation period is increased, cooling is observed throughout the majority of the ocean, including the deep ocean (Fig. 1). This drop in temperature peaks in the surface $1000 \mathrm{~m}$ of the equatorial regions. The only area of warming is near the surface at high latitudes (greater than $45^{\circ}$ ). The maximum cooling is $3^{\circ} \mathrm{C}$ and $6.2^{\circ} \mathrm{C}$ at 3 - and $10-$ day rotation periods, respectively. The warming observed is smaller in magnitude with values $0.6^{\circ} \mathrm{C}$ and $1.6^{\circ} \mathrm{C}$, respectively. Another effect of slowing the rotation is an increase in the strength of the overturning circulation. This is implied in the zonally averaged temperature profiles by the warming at the surface in areas of downwelling (the higher latitudes) and a cooling from depth throughout the lower latitudes where upwelling occurs. The latter results in a shallowing of the thermocline depth, which may be seen in the zonally averaged temperatures. Both of these behaviors are supported by the scaling analysis detailed below.

There is a clear and significant increase in the poleward heat transport when increasing the planetary rotation period (Fig. 2a). The peak heat transport increases from 1.5 to 

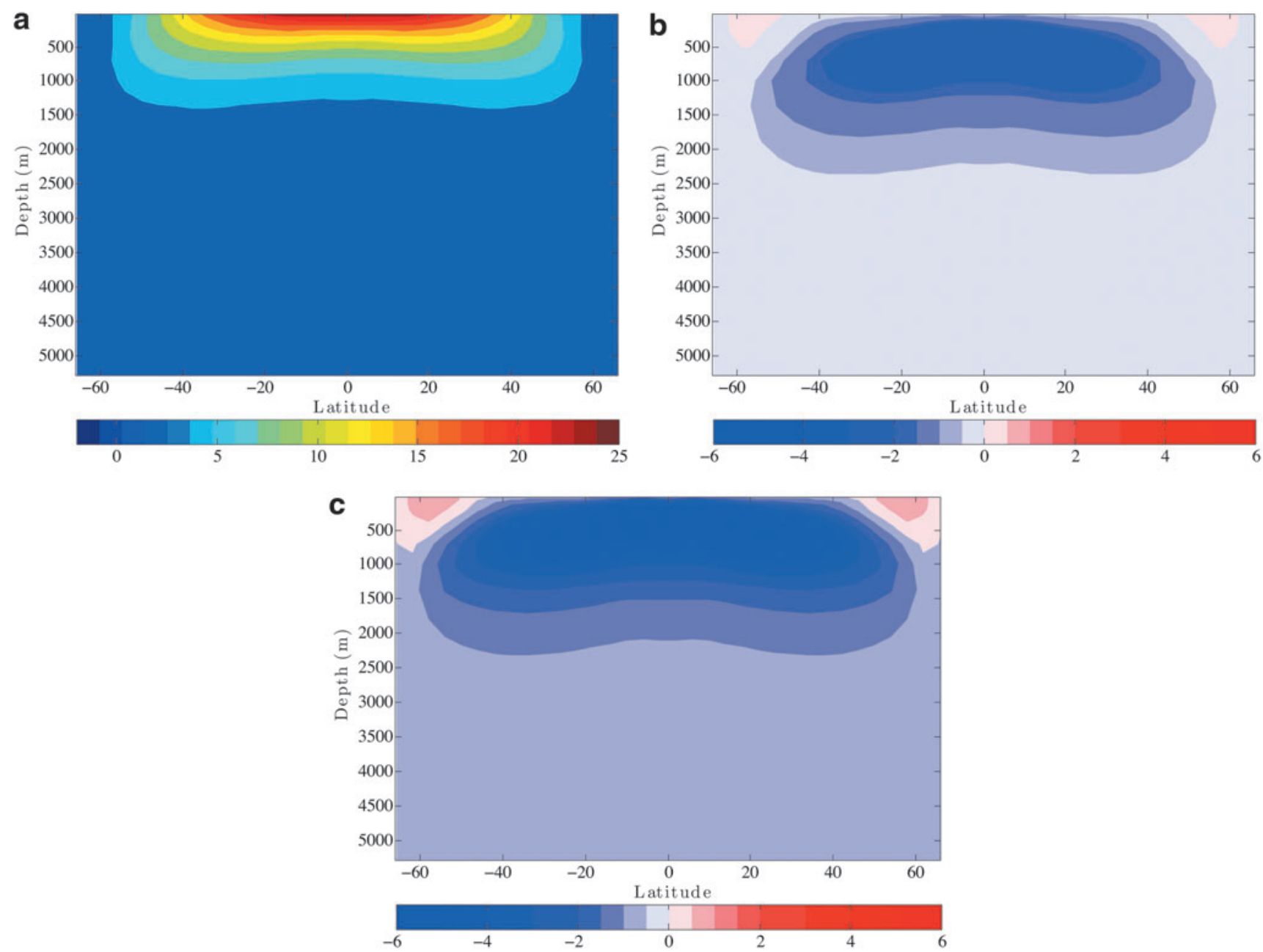

FIG. 1. (a) Zonally averaged temperature $\left({ }^{\circ} \mathrm{C}\right)$ for a ridgeplanet with 1 -day rotation period and no wind forcing. (b, c), The difference in the zonally averaged temperature $\left({ }^{\circ} \mathrm{C}\right)$ for a ridgeplanet with no wind forcing, from a 1- to 3-day rotation period and a 10-day rotation period, respectively. In each case, the data presented are an average of the final 1000 years of an 8000-year model run.

3.2 PW with an increase in the rotation period from 1 (light grey curve) to 10 (black curve) days. There is a significant difference in magnitude between the ridgeplanet runs with (dashed curve) and without (light grey curve) wind forcing. This is accounted for by the wind-driven Ekman component of the heat transport. The greater difference is between the Earth configuration (dotted curve) and wind-forced (dashed curve) ridgeplanet. The expected dependence of the ocean heat transport on the volume of the ocean is demonstrated by the significantly lower magnitude of transport in the northern hemisphere, where the ocean volume is considerably smaller for the Earth configuration. The latitude of the peak northward heat transport in the buoyancy-forced runs moves poleward with increasing rotation period. This behavior corresponds to an increase in the latitude at which the peak zonally averaged poleward velocity occurs in the upper ocean.

Due to the constraint of fixed surface forcing in the model, the magnitude of these changes in temperature is limited. Subsequently, a better measure of the potential impact of rotation period on the surface temperatures is the surface heat flux (Fig. 2b). As the rotation is slowed, there is an increase in the heat flux from the ocean to the atmosphere in the high latitudes. There is also an increase in the heat flux into the ocean, which occurs over a larger area, in the equatorial regions. Comparing the results from the runs with and without wind forcing at a 1-day rotation period shows that the wind forcing increases the magnitude of the flux of heat into the ocean in the equatorial region, but also restricts the area of heat flux into the ocean to a narrower equatorial region. The wind forcing has a smaller impact in the higher latitudes, which is where the rotation period has the largest impact on the surface heat flux. The impact of a longer rotation period is to decrease the equator-pole temperature gradient. The peak heat flux to the atmosphere moves poleward and increases, from 25 to $86 \mathrm{~W} \mathrm{~m}^{-2}$, when the rotation period is decreased from 1 to 10 days.

The impact of planetary rotation period on the ocean circulation, structure, and heat transport observed in the numerical results may be explained with a scale analysis of the governing equations (Bryan and Cox, 1967; Graham, 2008). The scales for the thermocline depth, $d$; the 
horizontal velocities, $U$; the overturning stream function, $\Psi$; and the heat transport, $Q$, are

$$
\begin{gathered}
d=\left(\frac{\kappa f L^{2}}{g \alpha \Delta T}\right)^{\frac{1}{3}}, \\
U=\left(\frac{\kappa(g \alpha \Delta T)^{2}}{L f^{2}}\right)^{\frac{1}{3}}, \\
\Psi=\left(\frac{\kappa^{2} g \alpha L^{4} \Delta T}{f}\right)^{\frac{1}{3}}, \\
Q=\rho_{0} C_{p}\left(\frac{\kappa^{2} g \alpha L^{4} \Delta T^{4}}{f}\right)^{\frac{1}{3}},
\end{gathered}
$$

where $\kappa$ is the thermal diffusivity, $f$ is the Coriolis parameter, $L$ is a horizontal length scale, $g$ is the gravitational constant, $\alpha$ is the coefficient of thermal expansion, $\Delta T$ is the equator-pole temperature gradient, $\rho_{0}$ is a reference density, and $C_{p}$ is the specific heat capacity. From the scalings (1)(4), the impact of varying the rotation rate $\Omega \sim f$ on the thermocline depth, horizontal velocities, overturning circulation, and heat transport is concluded to be

$$
d \sim \Omega^{\frac{1}{3}}, U \sim \Omega^{-\frac{2}{3}}, \psi \sim \Omega^{-\frac{1}{3}}, Q \sim \Omega^{-\frac{1}{3}} .
$$

Hence, this analysis allows the conclusion that a longer rotation period would result in a shallower thermocline, larger horizontal velocities, a stronger overturning circulation, and a greater poleward heat transport. The relations (5) obtained from the scale analysis confirm the behavior obtained from the numerical model. These scalings also predict values in the model results with surprising accuracy, for example, the peak ocean heat transport (Table 1).

\section{Discussion and Conclusion}

The results presented here show that the dependence of the poleward ocean heat transport on planetary rotation period is significantly greater than that of the atmosphere (Del Genio and Suozzo, 1987) at rotation periods longer than a day. The significance of the ocean transport at shorter rotation periods is also implied by the scale analysis detailed in this study. This highlights the importance of including this effect, through a dynamic ocean, to achieve more accurate modeling of the climates of terrestrial planets.

The range of rotation periods considered in this study corresponds to realistic exoplanetary rotation periods for not only Earth-like planets orbiting G-dwarf stars but also other types of stars, including M-dwarf stars. For very small Mdwarfs the rotation periods of tidally locked planets are less than 10 days, which is inferred from Tables 1 and 2 of Heath et al. (1999). For cases where the planet is not tidally locked, the rotation period is unknown and has the potential to be influenced by other factors such as additional planets in the system. Although only a restricted range of rotation periods have been analyzed in this study, the scaling analysis extends the presented argument to a much larger range
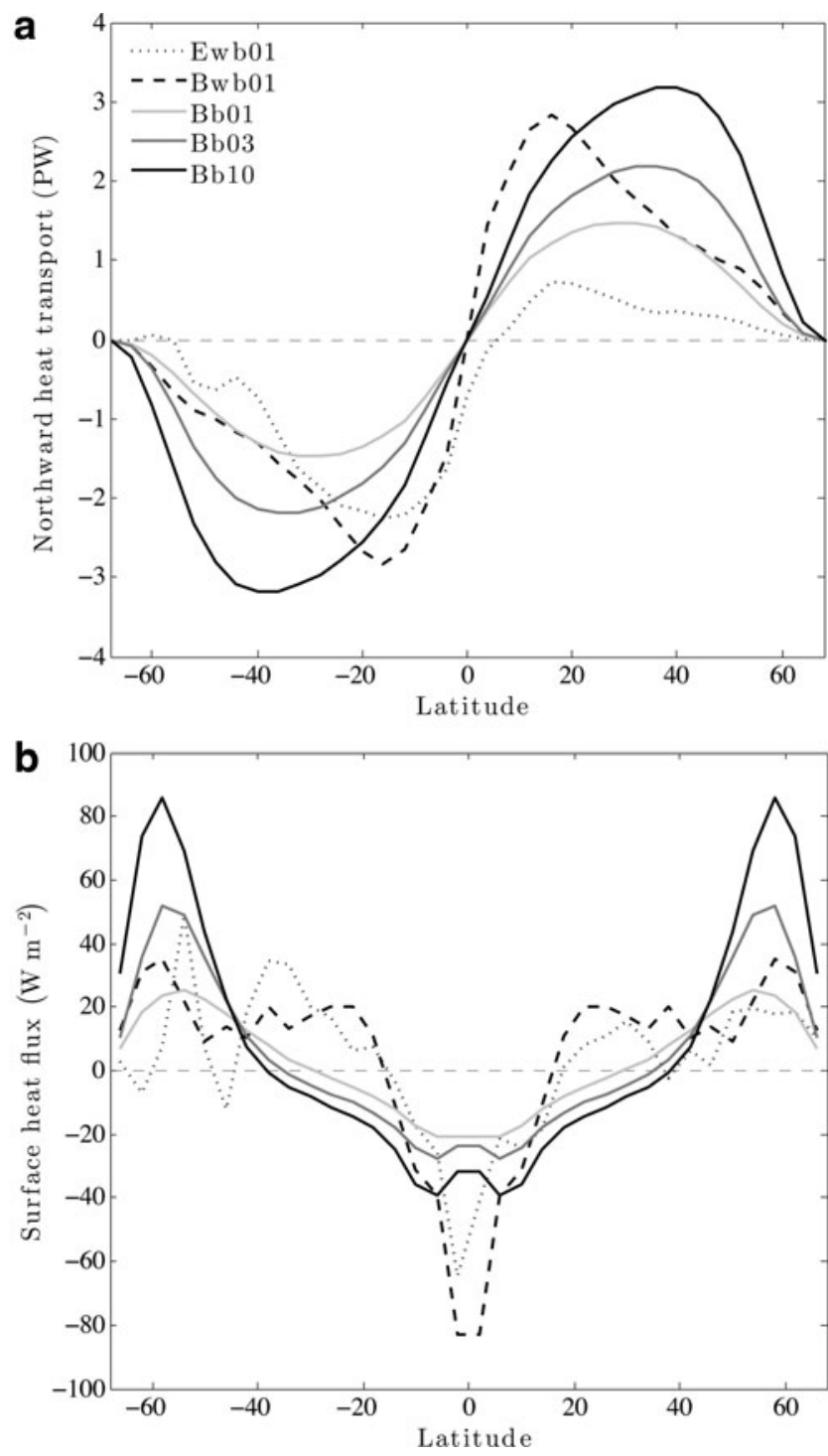

FIG. 2. (a) Northward ocean heat transport (PW) for Earth configuration (Ewb01), ridgeplanet at a 1-day rotation period with wind forcing (Bwb01) and with no wind forcing (Bb01), solely buoyancy-forced ridgeplanet at a 3-day rotation period (Bb03) and 10-day rotation period (Bb10). In each case, the data presented are an average of the final 1000 years of an 8000-year model run. (b) Zonally averaged surface heat flux $\left(\mathrm{W} \mathrm{m}^{-2}\right)$ for each of the configurations, using the same run notations as in (a). Positive heat flux is from the ocean to the atmosphere.

of rotation periods, both longer and shorter than that of Earth. The results presented also have significance for the terrestrial Archean climate, given the shorter rotation period, in addition to showing the importance of the ocean in the analysis of the habitability of both planets and moons.

In a wider context, the solubility of $\mathrm{CO}_{2}$ in water increases with decreasing temperature (Zeebe and Wolf-Gladrow, 2001). A planet having even an inorganic carbon cycle might have more $\mathrm{CO}_{2}$ dissolved in the ocean if it has a longer, rather than shorter, rotation period. For example, Archean Earth had a rotation period of approximately $12 \mathrm{~h}$ (Kiernet et al., 2013), which implies the potential for a warmer ocean. 
Table 1. Peak Ocean Heat Transport From the Model Compared to the Value Predicted by the Scaling $Q \sim \Omega^{-1 / 3}$

\begin{tabular}{llc}
\hline & \multicolumn{2}{c}{ Peak Q $(P W)$} \\
\cline { 2 - 3 } Rotation period (days) & Model & Scaling \\
\hline 1 & 1.478 & 1.478 \\
3 & 2.192 & 2.132 \\
10 & 3.178 & 3.184 \\
\hline
\end{tabular}

Scaling values are calculated using the model value at 1-day rotation period.

Wind forcing has been neglected to make the conclusions discussed here; however, with longer planetary rotation periods, the Ekman transport increases due to its dependence on the Coriolis parameter. Furthermore, atmospheric wind velocities increase as the rotation period is lengthened (Williams, 1988), which would only further increase the wind-driven poleward heat transport in the ocean. Hence, it would be expected that the component of the heat transport resulting from direct wind forcing would also increase with an increased rotation period, exaggerating the increase shown by the results presented. This only enhances the need for the ocean heat transport to be considered when investigating planetary climates.

To further this analysis requires the use of a coupled atmosphere-ocean model, which allows the combined dependence of both atmospheric and ocean heat transport on planetary rotation to be studied (e.g., Vallis and Farneti, 2009). There is also the possibility of investigating the case where there is no meridional boundary in the ocean by using an eddy-resolving ocean model, because it is expected that eddies would play a crucial role in the poleward heat transport, as discussed above. Other developments such as examining more realistic continental configurations or specific rotation periods would be contingent on the availability of future observations.

\section{Acknowledgments}

The research presented was carried out on the High Performance Computing Cluster supported by the Research and Specialist Computing Support service at the University of East Anglia. This work is funded by a studentship from the UK Engineering and Physical Sciences Research Council.

\section{Author Disclosure Statement}

No competing financial interests exist.

\section{References}

Akeson, R., Chen, X., Ciardi, D., Crane, M., Good, J., Harbut, M., Jackson, E., Kane, S., Laity, A., Leifer, S., Lynn, M., McElroy, D., Papin, M., Plavchan, P., Rez, S.R., Rey, R., von Braun, K., Wittman, M., Abajian, M., Ali, B., Beichman, C., Beekley, A., Berriman, G., Berukoff, S., Bryden, G., Chan, B., Groom, S., Lau, C., Payne, A., Regelson, M., Saucedo, M., Schmitz, M., Stauffer, J., Wyatt, P., and Zhang, A. (2013) The NASA exoplanet archive: data and tools for exoplanet research. arXiv:1307.2944.
Bryan, K. and Cox, M.D. (1967) A numerical investigation of the oceanic general circulation. Tellus 19:54-80.

Del Genio, A.D. and Suozzo, R.J. (1987) A comparative study of rapidly and slowly rotating dynamical regimes in a terrestrial general circulation model. Journal of the Atmospheric Sciences 44:973-986.

Edson, A., Lee, S., Bannon, P., Kasting, J.F., and Pollard, D. (2011) Atmospheric circulations of terrestrial planets orbiting low-mass stars. Icarus 212:1-13.

Graham, T. (2008) Sensitivity of the global climate to vertical ocean diffusivity and other parameters in coupled climate models. PhD thesis, University of East Anglia, Norwich, UK.

Hallberg, R. and Gnanadesikan, A. (2006) The role of eddies in determining the structure and response of the wind-driven Southern Hemisphere overturning: results from the Modeling Eddies in the Southern Ocean (MESO) project. J Phys Oceanogr 36:2232-2252.

Heath, M.J., Doyle, L.R., Joshi, M.M., and Haberle, R.M. (1999) Habitability of planets around red dwarf stars. Orig Life Evol Biosph 29:405-424.

Heller, R. and Armstrong, J. (2014) Superhabitable worlds. Astrobiology 14:50-66.

Hellerman, S. and Rosenstein, M. (1983) Normal monthly wind stress over the world ocean with error estimates. J Phys Oceanogr 13:1093-1104.

Hu, Y. and Yang, J. (2013) Role of ocean heat transport in climates of tidally locked exoplanets around M dwarf stars. Proc Natl Acad Sci USA 111:629-634.

Impey, C. (2013) The first thousand exoplanets: twenty years of excitement and discovery. In Astrobiology, History, and Society, Advances in Astrobiology and Biogeophysics, edited by D.A. Vakoch, Springer, Berlin, pp 201-212.

Joshi, M.M., Haberle, R.M., and Reynolds, R.T. (1997) Simulations of the atmospheres of synchronously rotating terrestrial planets orbiting $\mathrm{M}$ dwarfs: conditions for atmospheric collapse and the implications for habitability. Icarus 129: 450-465.

Kasting, J.F., Whitmire, D.P., and Reynolds, R.T. (1993) Habitable zones around main sequence stars. Icarus 101:108128.

Kiernet, H., Feulner, G., and Petoukhov, V. (2013) Albedo and heat transport in 3-D model simulations of the early Archean climate. Climate of the Past 9:1841-1862.

Levitus, S. (1982) Climatological Atlas of the World Ocean, NOAA Professional Paper 13, U.S. Department of Commerce, National Oceanic and Atmospheric Administration, U.S. Government Printing Office, Rockville, MD.

Merlis, T.M. and Schneider, T. (2010) Atmospheric dynamics of Earth-like tidally locked aquaplanets. Journal of Advances in Modeling Earth Systems 2, doi:10.3894/JAMES.2010.2.13.

Shields, A., Meadows, V., Bitz, C., Pierrehumbert, R., Joshi, M., and Robinson, T. (2013) The effect of host star spectral energy distribution and ice-albedo feedback on the climate of extrasolar planets. Astrobiology 13:715-739.

Smith, R. and Dubois, C. (2004) Ocean circulation and climate in an idealised Pangean OAGCM. Geophys Res Lett 31: L18207.

Smith, R.S., Dubois, C., and Marotzke, J. (2006) Global climate and ocean circulation on an aquaplanet ocean-atmosphere general circulation model. J Clim 19:4719-4737.

Trenberth, K.E. and Caron, J.M. (2001) Estimates of meridional atmosphere and ocean heat transports. J Clim 14:34333443. 
Vallis, G.K. and Farneti, R. (2009) Meridional energy transport in the coupled atmosphere-ocean system: scaling and numerical experiments. Quarterly Journal of the Royal Meteorological Society 135:1643-1660.

Webb, D.J. (1996) An ocean model code for array processor computers. Comput Geosci 22:569-578.

Williams, G.P. (1988) The dynamical range of global circulations-I. Clim Dyn 2:205-260.

Zeebe, R.E. and Wolf-Gladrow, D. (2001) $\mathrm{CO}_{2}$ in seawater: equilibrium, kinetics, isotopes. In Elsevier Oceanography Series, Vol. 65, Gulf Professional Publishing, Amsterdam.
Address correspondence to: Jodie Cullum School of Mathematics University of East Anglia Norwich, UK, NR4 7TJ

E-mail: J.Cullum@uea.ac.uk

Submitted 28 March 2014

Accepted 20 May 2014

This work is licensed under a Creative Commons Attribution 3.0 United States License. You are free to copy, distribute, transmit and adapt this work, but you must attribute this work as "Astrobiology. Copyright 2014 Mary Ann Liebert, Inc. http://liebertpub.com/ast, used under a Creative Commons Attribution License: http:// creativecommons.org/licenses/by/3.0/us/” 